\title{
Percolating through Networks of Random Thresholds: Finite Temperature Electron Tunneling in Metal Nanocrystal Arrays
}

\author{
Raghuveer Parthasarathy, ${ }^{1}$ Xiao-Min Lin, ${ }^{1,2}$ Klara Elteto, ${ }^{1}$ T. F. Rosenbaum, ${ }^{1}$ and Heinrich M. Jaeger ${ }^{1}$ \\ ${ }^{1}$ James Franck Institute and Department of Physics, University of Chicago, Chicago, Illinois 60637, USA \\ ${ }^{2}$ Materials Science Division and Chemistry Division, Argonne National Laboratory, Argonne, Illinois 60439, USA
}

(Received 17 February 2003; published 18 February 2004)

\begin{abstract}
We investigate how temperature affects transport through large networks of nonlinear conductances with distributed thresholds. In monolayers of weakly coupled gold nanocrystals, quenched charge disorder produces a range of local thresholds for the onset of electron tunneling. Our measurements delineate two regimes separated by a crossover temperature $T^{*}$. Up to $T^{*}$ the nonlinear zerotemperature shape of the current-voltage curves survives, but with a threshold voltage for conduction that decreases linearly with temperature. Above $T^{*}$ the threshold vanishes and the low-bias conductance increases rapidly with temperature. We develop a model that accounts for these findings and predicts $T^{*}$.
\end{abstract}

DOI: 10.1103/PhysRevLett.92.076801

Densely packed arrays of nanocrystals form a new class of "artificial solids" with tunable electronic, magnetic, and optical properties [1-7]. These properties stem from single-electron charging and quantum confinement energies on the individual particle level, mediated by the coupling to neighboring particles [8]. However, even for the simplest such artificial solid, a close-packed monolayer of metallic nanoparticles, the effect of temperature on the overall transport properties is poorly understood.

At low temperatures, metal nanoparticle arrays show pronounced nonlinear current-voltage $(I V)$ characteristics, with a well-defined threshold above which the applied bias has to be raised for conduction to occur. Similar threshold behavior occurs in a large family of disordered nonlinear systems, including the depinning of magnetic flux bundles in type-II superconductors [9] and the onset of motion in charge-density waves trapped by impurities [10]. In metal nanoparticle arrays the disorder arises, even for perfectly ordered particle arrangements, from quenched background charges in the substrate or the matrix surrounding the particles. These quenched charges induce random shifts in the local nanoparticle potentials and lead to a distribution of electrostatic energy costs incurred by electrons as they tunnel from particle to particle. The system behaves as a network of ultrasmall, Coulomb-blockade-type tunnel junctions [11] with a distribution of threshold energies. For $T=0$, a key result was obtained a decade ago by Middleton and Wingreen (MW) [12] who showed through simulations and a scaling model that the effective voltage threshold $V_{t}$ is proportional to the array length and that the current-voltage $(I V)$ characteristics exhibit power law scaling of the total current, $I$, with excess voltage, $V-V_{t}$. These predictions are in line with experimental results on a wide range of nanocrystal arrays $[1,4,5,7,8]$. More recently, the effect of structural disorder on the scaling behavior was investigated by experiments and reproduced by simulations $[7,13,14]$.
PACS numbers: 73.22.-f, 05.60.Gg, 73.21.Cd, 73.40.Gk

For finite temperatures, on the other hand, the literature to date shows no consensus. In single-particle systems the shape of the $I V$ curves near threshold is exponentially sensitive to temperature [11]. For arrays, Heath's group reported that increasing temperature rapidly rounds out any nonlinearities $[5,8,15]$. Black et al. also observed linear behavior above $40 \mathrm{~K}$ [1]. Other recent work found that finite temperature had a much weaker effect on the shape of the $I V$ characteristics and on the threshold $V_{t}$ $[7,14,16,17]$. Theoretical approaches that could calculate temperature-dependent $I V$ s for large arrays are currently not available.

To address this issue, we have performed systematic experiments on large, well-characterized twodimensional arrays of gold nanocrystals. Our results show that the $I V$ curves retain their low- $T$, nonlinear scaling properties to remarkably high temperatures and that $V_{t}$ decreases only linearly with $T$. We can explain these findings by assuming that there is a subset of junctions, percolating across the array, for which thermal fluctuations effectively have removed the Coulomb blockade. The starting point is the picture developed by MW of charge fronts propagating through the array [12]. However, our model goes beyond MW in three key aspects: it introduces a way to deal with the effect of temperature on a distribution of charging energies, it goes beyond square lattices and applies to arbitrary regular networks, and it can explicitly take into account capacitive coupling between neighboring islands, as is appropriate for close-packed arrays. We believe that this very general approach may also be applicable to other systems with distributions of local threshold values.

Our arrays consisted of monolayers of 1dodecanethiol-ligated gold nanocrystals, synthesized and deposited from solution onto silicon nitride "window" substrates [18,19]. Several arrays were deposited onto substrates with thicker $\mathrm{Si}_{3} \mathrm{~N}_{4}$ layers and no windows, 
more suitable for measurements above $200 \mathrm{~K}$. Eleven arrays were studied ranging from monolayers with long-range hexagonal order, with domains of typical size equal to the electrode spacing, to monolayers with some disorder, including voids or localized regions of double layers not more than $20 \%$ in area fraction. In each sample, the nanocrystals were monodisperse to within $5 \%$ as checked by transmission electron microscopy (TEM). Different samples had mean core diameters $4.5<d<6.5 \mathrm{~nm}$ and mean spacings $1.5<s<2.6 \mathrm{~nm}$. The relevant Coulomb charging energy, $\Delta E$, is the change in electrostatic energy of the system due to an electron tunneling from one particle to the next. $\Delta E$ depends on the background charges on all particles involved. However, because of compensation by mobile electrons, it suffices to consider effective background charges within $[-e / 2,+e / 2]$ and the distribution of charging energies, $P(\Delta E)$, extends only to $\pm \Delta E_{\max }$. For closepacked monolayers, keeping only interactions between the two particles forming the junction and the surrounding eight nearest neighbors, to good approximation $\Delta E_{\max }=e^{2}\left(\mathbf{C}_{11}^{-1}+2 \mathbf{C}_{12}^{-1}\right)=e^{2} \mathbf{C}_{11}^{-1}(1+2 \gamma)$, with $\gamma=$ $\mathbf{C}_{12}^{-1} / \mathbf{C}_{11}^{-1}$, where $\mathbf{C}^{-1}$ is the inverse capacitance matrix. From measurements of $d$ and $s$, and taking a dielectric constant $\epsilon=2$ for the alkanethiol, the inverse capacitance matrix elements for such a ten-particle system were calculated numerically [20]. This gave $\mathbf{C}_{11}^{-1}$ of order $10^{18} \mathrm{~F}^{-1}$ and typical values for $\gamma \approx 0.36$. All arrays had a fixed width $(2 \mu \mathrm{m}$, or $\approx 270$ particles $)$ and lengths, $N$, ranging from 27-170 particles, set by the electrode separation. For samples on window substrates, $N$ was measured by direct inspection with TEM after the transport measurements were completed. Otherwise, $N$ was estimated from the electrode separation. Samples were cooled in vacuum, and dc $I V$ curves were taken using Keithley 614 electrometers at voltage ramp rates $5-25 \mathrm{mV} / \mathrm{s}$.

At low temperatures, all arrays showed a clear voltage threshold for conduction, indicating strong Coulomb blockade behavior. Close inspection of the $I V$ curves (see Fig. 1) reveals that the differential conductance, $g \equiv d I / d V$, for a given current $I$ is independent of temperature below a sample-dependent characteristic temperature in the range $100-200 \mathrm{~K}$. The $I V$ curves keep their shape and simply shift to lower voltages with increasing temperature. More quantitatively, we find that the $I V$ s can be collapsed on top of each other by translating them by an amount $V_{\text {shift }}(T)<0$. Defining an effective threshold $V_{t}(T)=V_{t}(0)+V_{\text {shift }}(T)$, we can collapse all traces onto a master curve as shown in Fig. 1(b). $\left|V_{\text {shift }}\left(T^{*}\right)\right|=V_{t}(0)$ defines the temperature, $T^{*}$, at which the array threshold first reaches zero. Once $T>T^{*}$, the $I V$ s exhibit nonzero differential conductance at zero bias [Fig. 1(a) inset] prohibiting a data collapse at small bias. However, at higher bias the $I V$ s still collapse when translated by an amount $\left|V_{\text {shift }}(T)\right|>V_{t}(0)$. The regime above $T^{*}$ thus is characterized by a negative effective threshold and low-bias data falling below the master curve.

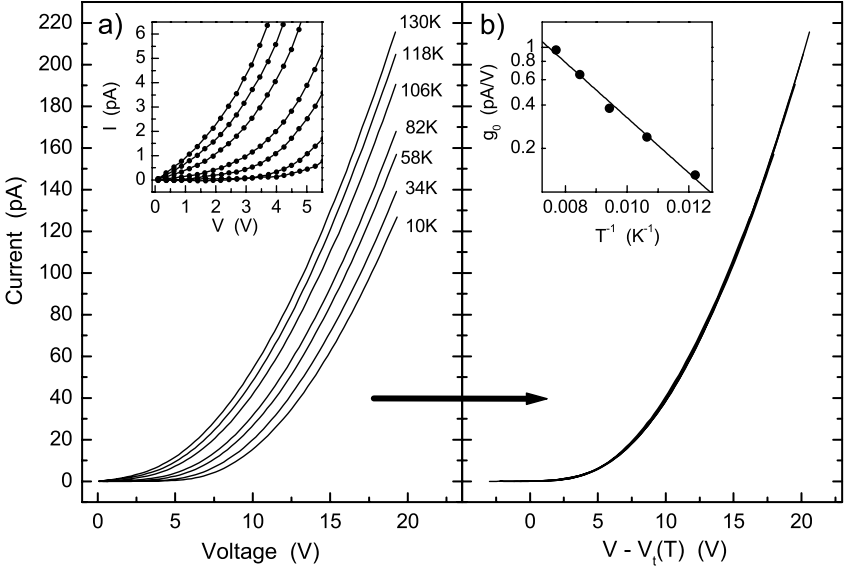

FIG. 1. (a) Evolution of $I V$ curves with temperature for an array of length $N=128$. The $I V$ s are fully symmetric with respect to bias voltage reversal. Above $T^{*}$ the $I V \mathrm{~s}$ no longer have a finite voltage threshold, as shown in the inset. (b) Below $T^{*}, I V \mathrm{~s}$ at different temperatures can be collapsed by translating the voltage scale by an amount $V_{\text {shift }}(T)$, with no alteration of the current scale. The inset shows the zero-bias conductance, $g_{0}=d I / d V$, as a function of temperature.

For highly ordered arrays [bottom curve in Fig. 2(a)] MW's $T=0$ scaling law, $I \sim\left(V-V_{t}\right)^{\zeta}$, remains valid up to $T^{*}$ with temperature-independent exponent $\zeta=2.25 \pm$ 0.1 . Details of the functional form of $I(V)$ in different arrays affect the value of $T^{*}$ but are irrelevant as far as the collapse is concerned. Even for slightly disordered arrays, characterized by a somewhat $s$-shaped $I V$ [7] in Fig. 2, collapse can be achieved. For all arrays studied, $V_{t}(T)$, the only adjustable parameter in the scaling procedure drops essentially linearly with increasing temperature [Fig. 2(b)]. Both $V_{t}(0)$ and the slope of this
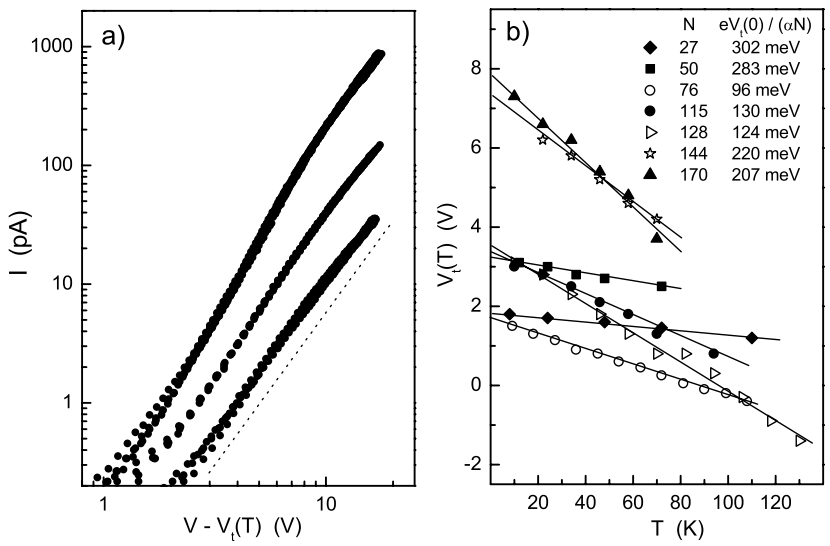

FIG. 2. (a) Scaling behavior of current $I$ with excess voltage $V-V_{t}(T)$ above threshold for the same array as in Fig. 1 (middle curve) and two other arrays with different degrees of disorder. For each array, four different temperatures are plotted. The dotted line corresponds to $\zeta=2.27$. (b) The effective threshold as a function of temperature for several arrays of length $N$ and effective charging energy $e V_{t}(0) / \alpha N$. 
depression increase with array size $N$. Furthermore, for similar $V_{t}(0)$ the slope decreases with increasing charging energy.

This slow, linear depression of the voltage threshold with temperature is surprising and unexpected from the theory of few-junction networks [11] or from other nonlinear systems such as charge-density waves [10]. A similar trend was first noted in 1D chains of carbon nanospheres by Bezryadin et al. [16] and by Ancona et al. [14] in small 2D arrays. Bezryadin et al. proposed that thermal fluctuations reduce the charging energy by $k_{B} T$ in all particles and that therefore $V_{t}(T)$ should decrease as $N k_{B} T / e$. However, this simple picture cannot account for the slope values in Fig. 2(b). We now outline a model that captures the linear dependence of $V_{t}(T)$, predicts the slope, and, under appropriate rescaling, collapses the data in Fig. 2 onto a universal curve.

MW showed that the minimum bias voltage for charges to move all the way from one electrode to the other depends on the number of "up-steps" along any given path. Each local up-step in potential, no matter how small, requires an increment $\Delta V$ in the externally applied bias for the charge front to advance, and thus contributes equally. ( $\Delta V=\Delta E_{\max } / e$ for uncoupled particles or junctions surrounded by charge neutral neighbors; for the more general case, see below.) The array threshold is set by the optimal path with the smallest total number of upsteps and scales as $V_{t}=\alpha N \Delta V$ in both 1D and 2D [12]. In $2 \mathrm{D}, \alpha=0.338$ for square lattices [12] and $\alpha=0.227$ for triangular lattices [21]. Down-steps do not gain energy because all tunneling between particles is inelastic (no resonant tunneling between sharp levels). Hence, as long as the number of up-steps along a path remains fixed, a gradual reduction in the associated energy step size, induced by thermal fluctuations, will not reduce $V_{t}$. We now argue that $V_{t}$ can be reduced if temperature eliminates some of the up-steps completely. This occurs for the subset of junctions with step magnitudes $|\Delta E|$ so small that they are washed out by thermal fluctuations.

We consider a scenario in which temperature has removed the nonlinear, threshold-type Coulomb blockade behavior of a fraction $p(T)$ of the junctions (irrespective of the sign of the step), effectively turning them into linear resistors. The effect of temperature, then, is a shift in threshold proportional to $p(T)$. At some temperature, $T^{*}$, the fraction $p\left(T^{*}\right)$ will become large enough for a continuous path of junctions without discernible Coulomb blockade to form a percolating subnetwork that bridges the array. At this point the overall array voltage threshold will vanish. We therefore identify $p\left(T^{*}\right)$ with the percolation threshold, $p_{c}$, for the underlying lattice. Hence we expect $V_{t}(T)=V_{t}(0)\left[1-p(T) / p_{c}\right]$. For a triangular lattice $p_{c} \approx 0.347$. To make explicit the temperature dependence of the threshold voltage, we need to find $p(T)$. For a given distribution, $P(\Delta E)$, junctions corresponding to $|\Delta E|<b k_{B} T$ are taken as effectively linearized. The factor $b$ accounts for the broadening of the Fermi-Dirac distributions in the nanocrystals. Electrons in the highenergy tail of one nanocrystal's distribution can tunnel into the low-energy tail of accessible states of a neighboring particle, reducing the energy cost by roughly $2 k_{B} T$. More accurately, equating the reduction with the difference between the mean energies in the high- and lowenergy tails, we calculate $b=2.4$ [21]. At any given temperature, $p(T)$ is then given by the integral over $P(\Delta E)$ between the limits $\pm b k_{B} T$.

Consider first the case of two particles with random charge offsets in the range $[-e / 2, e / 2]$, forming a representative tunnel junction, surrounded by eight neutral neighbors. In this case, $\Delta E_{\max }=e^{2} \mathbf{C}_{11}^{-1}(1-\gamma)$, and $P(\Delta E)$ is a triangle with $y$ intercept $P(0)=1 / \Delta E_{\max }$ (inset to Fig. 3) [21]. Integrating $P(\Delta E)$ over the shaded area leads to $p(T)=b x(2-b x)$ with $x=k_{B} T P(0)$. Using $p\left(T^{*}\right)=p_{c}$ and $b=2.4$ we find $k_{B} T^{*} \approx$ $0.08 / P(0)$. Note that $p(T)$ is proportional to $T$ and $P(0)$ as long as $x \ll 1$, which is satisfied up to temperatures far above $T^{*}$. Allowing for random offset charges also on the eight neighbors rounds the corners and steepens the slope of $P(\Delta E)$. Still, the overall triangular shape remains, $p(T)=2 b x+\mathcal{O}\left(x^{3}\right)$, and in the above considerations only the numerical value for $P(0)$ needs to be modified. Now $\Delta E_{\max }=e^{2} \mathbf{C}_{11}^{-1}(1+2 \gamma)$ and $P(0)=$ $\left(e^{2} \mathbf{C}_{11}^{-1}\right)^{-1}(1-1.57 \gamma) /(1-\gamma)^{2}$ [21]. For all arrays, independent of $N$, the model then predicts that the normalized threshold $V_{t}(T) / V_{t}(0)$ decreases linearly as a function of the dimensionless variable $x=k_{B} T P(0)$, with a slope $-2 b / p_{c}$, crossing zero near $x=0.072$. Within this picture, $1 / P(0)$ can be thought of as an effective charging energy per up-step that captures all effects of the local packing geometry and the quenched charge distribution.

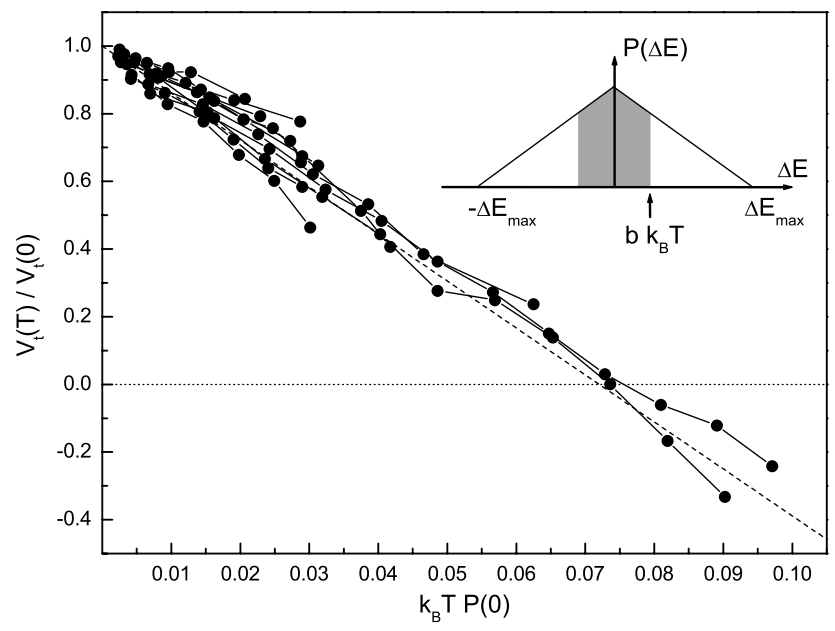

FIG. 3. Normalized threshold, $V_{t}(T) / V_{t}(0)$ as a function of the dimensionless temperature variable $x=k_{B} T P(0)$ for all arrays. The dashed line is the model prediction. Inset: probability density of charging energies $P(\Delta E)$. With capacitive coupling in the presence of offset charges on all nearest neighbors, $P(\Delta E)$ evolves to a more rounded shape. 
These predictions agree well with our data (Fig. 3) [22]. To obtain $V_{t}(0)$ we extrapolated $V_{t}(T)$ back to $T=0$ for each sample [Fig. 2(b)]. To determine $P(0)$ two methods were used, giving independent checks of the model. Equating $e \Delta V=1 / P(0)$ for the energy per up-step we obtained $P(0)$ from $V_{t}(0)=\alpha N \Delta V$ using $N$ from the electrode separations and $\alpha=0.227$. In addition, for the samples where TEM images of sufficient resolution were available to extract $d$ and $s$, we were able to crosscheck the values for $P(0)$ with those derived from direct numerical calculation of the $\mathbf{C}_{i j}^{-1}$ [20].

The broad distribution of local energy differences $\Delta E$ guarantees that, even if a fraction of steps is effectively removed, the underlying concept of current paths meandering through a random potential landscape - the premise of MW's scaling picture - remains valid. The general shape of the $I V \mathrm{~s}$ above $V_{t}(T)$, which reflects how additional current paths open up with increasing bias beyond threshold, therefore should remain unchanged up to $T^{*}$. This is borne out by our data. Rounding of the Coulomb blockade in the local junction $I V$ characteristics affects the quality of the data collapse only at the smallest $V-$ $V_{t}(T)$ [Fig. 2(a)].

The behavior for $T>T^{*}$ is quite different. Here the low-bias conductance is dominated by a few junctions from the $\left(p_{c} / 2\right)$ th percentile of $P(\Delta E)$, since they are the key, "bottleneck" bonds in the lattice during percolation at $T^{*}$. Therefore, the low-bias $I V \mathrm{~s}$ for the percolating path resemble those of a single island system with washed-out Coulomb blockade, for which the linear zero-bias conductance [Fig. 1(a) inset] exhibits thermally activated behavior [11]: $g_{0}(T) \sim \exp \left(-U / k_{B} T\right)$. Our model predicts $U=b k_{B} T^{*}=0.17 \Delta E_{\max }$, the largest $\Delta E$ for the percolating path. This $g_{0}$ will dominate the low-bias array conductance as $T$ increases and additional, parallel paths open up that have larger $U$. Because of these additional paths, we expect the measured effective activation energy to be somewhat larger than $b k_{B} T^{*}$. Our data indeed are compatible with simple activated behavior and a magnitude of $U 1$ to 2 times $b k_{B} T^{*}$. For example, for the array in the Fig. 1(b) inset $\Delta E_{\max }=124 \mathrm{meV}$, giving $b k_{B} T^{*}=$ $21 \mathrm{meV}$, to be compared with $U \approx 38 \mathrm{meV}$ from the slope of $\ln \left(g_{0}\right)$ vs $T^{-1}$. However, because of the limited (high) temperature range available, our data cannot rule out other functional forms, such as variable range hopping conduction as seen in Ref. [15].

Our results support a picture of thermally assisted quantum tunneling between neighboring gold nanocrystal cores through the dodecanethiol ligand. Within this picture, the ligands serve as a mechanical spacer between the particles, and they set the tunneling barrier height. They do not introduce additional states into the barrier, nor is the barrier low enough to be thermally hopped over [23], both of which may be the case with more complex ligands [4]. We find no evidence of a regime with a positive temperature coefficient of resistance as seen in Ref. [5]. Such behavior may require a stronger interpar- ticle coupling and junction resistances much closer to the quantum resistance $R_{q}=h / e^{2}$. In the weak coupling limit, as in our arrays, the value of $T^{*}$ as well as the collapse of the temperature-dependent threshold data onto a universal curve emerges naturally from considering the interplay of tunneling and quenched charge disorder.

We thank Qiti Guo and Benjamin Lauderdale for experimental assistance and Eduard Antonyan, Terry Bigioni, Ilya Gruzberg, Alan Middleton, Toan Nguyen, and Tom Witten for stimulating discussions. X.-M. L. acknowledges support from DOE W-31-109-ENG-38. This work was supported by the UC-ANL Consortium for Nanoscience Research and by the NSF MRSEC program under DMR-0213745.

[1] C. T. Black, C. B. Murray, R. L. Sandstrom, and S. Sun, Science 290, 1131 (2000).

[2] R. C. Doty, H. Yu, C. K. Shih, and B. Korgel, J. Phys. Chem. B 105, 8291 (2001).

[3] C. A. Leatherdale et al., Phys. Rev. B 62, 2669 (2000).

[4] R. P. Andres et al., Science 273, 1690 (1996).

[5] J. F. Sampaio, K. C. Beverly, and J. R. Heath, J. Phys. Chem. B 105, 8797 (2001).

[6] D. S. Ginger and N. C. Greenham, J. Appl. Phys. 87, 1361 (2000).

[7] R. Parthasarathy, X.-M. Lin, and H. M. Jaeger, Phys. Rev. Lett. 87, 186807 (2001).

[8] C. P. Collier, T. Vossmeyer, and J. R. Heath, Annu. Rev. Phys. Chem. 49, 371 (1998).

[9] S. Bhattacharya and M. J. Higgins, Phys. Rev. Lett. 70, 2617 (1993).

[10] G. Grüner, Rev. Mod. Phys. 60, 1129 (1988).

[11] Single Charge Tunneling, edited by H. Grabert and M. H. Devoret (Plenum, New York, 1992).

[12] A. A. Middleton and N. S. Wingreen, Phys. Rev. Lett. 71, 3198 (1993).

[13] C. Reichhardt and C. J. Olson Reichhardt, Phys. Rev. Lett. 90, 046802 (2003).

[14] M. G. Ancona et al., Phys. Rev. B 64, 033408 (2001).

[15] K. C. Beverly, J. F. Sampaio, and J. R. Heath, J. Phys. Chem. B 106, 2131 (2002).

[16] A. Bezryadin et al., Appl. Phys. Lett. 74, 2699 (1999).

[17] C. Reichhardt and C. J. Olson Reichhardt, Phys. Rev. B 68, 165305 (2003).

[18] X.-M. Lin et al., J. Nanopart. Res. 2, 157 (2000).

[19] X.-M. Lin, H. M. Jaeger, C. M. Sorensen, and K. J. Klabunde, J. Phys. Chem. B 105, 3353 (2001).

[20] FASTCAP capacitance calculator program, MIT, (C) 1992.

[21] For details about these calculations as well as supporting simulations, see K. Elteto et al. (to be published).

[22] In $1 \mathrm{D} p_{c}=1$ and $\alpha=1 / 2$, and our model predicts $V_{t}(T)=V_{t}(0)[1-p(T)] \approx V_{t}(0)-1.2 N k_{B} T / e, \quad$ reproducing the behavior observed in [16] for nanoparticle chains.

[23] R. L. Lingle et al., Chem. Phys. 205, 191 (1996); 208, 297 (1996).

$076801-4$ 